Letters to the Editor

\section{Angiotensin converting enzyme inhibitors for hypertension and heart failure?}

Sir,

The title of Cleland's leading article ${ }^{1}$ wisely ended with a question mark rather than a period. In the current enthusiasm for angiotensin converting enzyme (ACE) inhibitors for congestive heart failure ${ }^{2}$ Cleland cautioned that 'used for the wrong patient or in the wrong situation, these drugs also have the power to do harm.' His warning is particularly appropriate in view of recent information concerning their use in pregnant women.

The SOLVD (Studies of Left Ventricular Dysfunction) study which compared enalapril with conventional therapy has two arms. Although the results of the treatment study reported last November at the American Heart Association annual meeting showed a highly significant $16 \%$ reduction in overall mortality during an average follow-up period of 41.4 months, there was no significant decrease in mortality with enalapril at 48 months in the prevention arm, the results of which were reported at the American College of Cardiology annual meeting in April 1992.

Therefore, studies like SOLVD, SAVE (Survival and Ventricular Enlargement), CONSENSUS (Cooperative North Scandinavian Enalapril Survival Study), CONSENSUS II, V-HeFT II (Second Veterans Administration Heart Failure Trial), ISIS-4 (Fourth International Study of Infarct Survival) and AIRE (Acute Infarction Ramipril Efficacy) with different ACE inhibitors all raise new hopes for patients with congestive heart failure, but the problem is by no means solved.

Professor Tsung $\mathrm{O}$. Cheng The George Washington University Medical Center, Division of Cardiology, 2150 Pennsylvania Ave., N.W., Washington, DC 20037, USA.

\section{References}

1. Cleland, J.G.F. Angiotensin converting enzyme inhibitors for hypertension and heart failure? Postgrad Med J 1992, 68: 1-5.

2. Cheng, T.O. Congestive heart failure in coronary artery disease. Am J Med 1991, 91: 409-415.

\section{Thyroid register audit: a district general hospital experience}

Sir,

Computerized thyroid registers were first introduced in the $1960 \mathrm{~s}^{1}$ so that suitable patients could be monitored regularly, usually by annual recall by the computer for thyroid function tests (TFT). This obviated the need for attendance at the endocrine clinics unless an abnormal thyroid function test was detected. Since then, several studies have been published ${ }^{1-4}$ confirming thyroid registers to be both efficient and cost effective with a saving of up to $40 \%$ compared to the costs of regular hospital follow-up. Despite this, follow-up via computerized registers remains available in only a few regional centres.

With the high prevalence of thyroid disease and the need for long-term follow-up, the increasing size of thyroid registers could have a negative effect on the proven efficiency of these registers. District general hospitals could therefore help by setting up their own computerized thyroid registers.

We set up our own in 1987, which we believe is the first of its kind in a district general hospital in the UK. An audit of our 3 year experience with the 132 patients in the register confirmed that it was efficient and successful in recalling all the patients except for $3 \%$ who were lost to follow-up. All results were sent to the general practitioner, together with suggestions such as the need for repeats if the results were abnormal. Unfortunately, most of the patients with abnormal thyroid stimulating hormone (TSH) levels did not have repeat TFT as suggested, and this problem remained undetected for some time. This could conceivably be a problem with other thyroid registers, especially without the added scrutiny of an on-going clinical trial. We have therefore incorporated an 'alarm system' into the computer whereby patients who have not had repeat TFT within 6 weeks of an abnormal TSH would automatically be recalled to the hospital clinic.

Alternatively, monthly review of TFTs from the thyroid register could be undertaken, as in some centres, and patients recalled if necessary. However, this is more time-consuming, especially with large registers, and sub: ject to operator variability and errors. It will also fail to make maximum use of the computer. Therefore the name of the referring consultant and the address of the hospital clinic should also be entered into the computer so that the patient can be recalled if necessary to the appropriate clinic. The patient category according to diagnosis and treatment should also be entered into the computer, as it will in the future represent a valuable source of epidemiological information. This has been used in the SAFUR programme which showed no association between thyroid disease and breast cancer. ${ }^{5}$

With the high prevalence of thyroid disease and the accepted need for long-term follow-up, the future savings to the health service must be considerable if computerized thyroid registers were to be introduced nationally to include district general hospitals. This will not be at the expense of patient care as, in fact, computerized thyroid registers have been found to be more efficient, whilst providing a more uniform and consistent level of surveillance especially for patients dispersed over a wide geographical area, and have a loss to follow-up rate of three times less than that of hospital follow-up clinics. ${ }^{3}$ In our experience, the logistics of setting up a local thyroid register have been fairly simple, using a computer system in the Clinical Chemistry Laboratory that should be readily available, if not already installed, in many hospitals. The advantages and success of thyroid registers are well documented and our experience suggest that similar registers can be readily set up in district general hospitals with minimal expense. We also suggest incorporating a safety measure into all registers to screen out patients with 